\title{
HEAT KERNEL AND MODULI SPACE
}

\author{
KeFEng LiU
}

\section{Introduction and notations}

In this paper we describe a proof of the formulas of Witten [W1], [W2] about the symplectic volumes and the intersection numbers of the moduli spaces of principal bundles on a compact Riemann surface. It is known that these formulas give all the information needed for the Verlinde formula. The main idea of the proof is to use the heat kernel on compact Lie groups, in a way very similar to the heat kernel proof of the Atiyah-Singer index formula and the Atiyah-Bott fixed point formula. The Reidemeister torsion comes into the picture, through a beautiful observation of Witten, as the symplectic volume of the moduli space. It plays the role similar to that played by the Ray-Singer torsion in the path-integral computations on the space of connections.

The basic idea is as follows. Consider a smooth map between two compact smooth manifolds $f: M \rightarrow N$. Let $H\left(t, x, x_{0}\right)$ be the heat kernel of the Laplace-Beltrami operator on $N$ with $x_{0}$ a fixed regular value of $f$. Because of the basic properties of the heat kernel, we know that for any continuous function $a(y)$ on $M$, when $t$ goes to zero,

$$
\int_{M} a(y) H\left(t, f(y), x_{0}\right) d y=\int_{f^{-1}\left(B_{\delta}\left(x_{0}\right)\right)} a(y) H\left(t, f(y), x_{0}\right) d y+O\left(e^{-\delta / t}\right)
$$

where $B_{\delta}$ is a ball of radius $\delta$ around $x_{0}$, that is, the integral is localized to the neighborhood of $f^{-1}\left(x_{0}\right)$.

On the other hand let $\left\{\phi_{j}(x)\right\}$ be the orthonormal basis of the eigenvectors of the Laplace-Beltrami operator on $N$, then $H\left(t, x, x_{0}\right)$ has an expression

$$
H\left(t, x, x_{0}\right)=\sum_{j} e^{-\lambda_{j} t} \phi_{j}(x) \phi_{j}\left(x_{0}\right)
$$

Received July 23, 1996.

This work has been partially supported by an NSF grant. 
where $-\lambda_{j}$ is the eigenvalue of $\phi_{j}(x)$. So we have

$$
\begin{gathered}
\int_{f^{-1}\left(B_{\delta}\left(x_{0}\right)\right)} a(y) H\left(t, f(y), x_{0}\right) d y+O\left(e^{-\delta / t}\right)= \\
\sum_{j} e^{-\lambda_{j} t} \phi_{j}\left(x_{0}\right) \int_{M} a(y) \phi_{j}(f(y)) d y .
\end{gathered}
$$

This simple formula about heat kernel, which is just a general Poisson summation formula, can be viewed as a generalized version of the modular transformation formula for the classical theta-functions. The method in this paper to prove the formulas of Witten about moduli spaces will be based on this simple fact. We believe that such method should have more applications. For example, when $N$ is noncompact, we can easily derive a similar formula to (0), which, applied to the moment map, gives the nonabelian localization formula in symplectic geometry.

Now we fix some notations to be used in this paper. Let $G$ be a semisimple simply connected compact Lie group, $\mathcal{G}$ be its Lie algebra. Let $T$ be a maximal torus in $G$ and $\mathcal{T}$ be its Lie algebra. Let $\Delta \in \mathcal{T}^{*}$ be the root system of the complexification of the Lie algebra of $G, \mathcal{G}^{C}$, with respect to $\mathcal{T}$. Fix a Weyl chamber $\mathcal{C} \subset \mathcal{T}$ and let $\triangle^{+}$be the set of positive roots. Let $\Pi=\left\{\alpha_{1}, \cdots, \alpha_{l}\right\}, l=\operatorname{dim} \mathcal{T}$ be a fundamental system of $\Delta$. The Killing form induces a biinvariant metric $\langle\cdot, \cdot\rangle$ on $G$, this will be the biinvariant metric to be used in this paper. We identify $\mathcal{T}$ with $\mathcal{T}^{*}$ under which for any $\lambda \in \mathcal{T}^{*}$, there is a unique element $H_{\lambda} \in \mathcal{T}$ such that $<H_{\lambda}, H>=\lambda(H)$ for any $H \in \mathcal{T}$.

Introduce

$$
\lambda^{v}=\frac{2}{<\lambda, \lambda>} \lambda, H_{\lambda}^{v}=\frac{2}{<\lambda, \lambda>} H_{\lambda},
$$

and let

$$
\Gamma=2 \pi \sum_{j=1}^{l} Z H_{\alpha_{j}}^{v}=\{H \in \mathcal{T} ; \exp H=e\},
$$

and

$$
I=\left\{\lambda \in \mathcal{T}^{*} ; \lambda(\Gamma) \in 2 \pi Z\right\} .
$$

Put

$$
P_{+}=\left\{\lambda \in I ;<\lambda, \alpha_{j}>\geq 0,1 \leq j \leq l\right\}
$$

as the dominant integral weights.

Define $\lambda_{i} \in \mathcal{T}^{*}$ by $<\lambda_{i}, \alpha_{j}^{v}>=\delta_{i j}, 1 \leq i, j \leq l$, then

$$
I=\sum_{j=1}^{l} Z \lambda_{j}, \text { and } P_{+}=\left\{\sum_{j=1}^{l} m_{j} \lambda_{j} ; m_{j} \geq 0, m_{j} \in Z\right\} .
$$


There is a one-one correspondence between $P_{+}$and the equivalence classes of irreducible representations of $G$. For $\lambda \in P_{+}$, we let $\chi_{\lambda}$ and respectively $d_{\lambda}$ be the character and dimension of the irreducible representation corresponding to $\lambda$. Let $e$ be the identity element in $G$, then one has

$$
\chi_{\lambda}(e)=d_{\lambda}=\prod_{\alpha \in \Delta^{+}} \frac{\langle\lambda+\rho, \alpha>}{\langle\rho, \alpha>},
$$

where $\rho=\frac{1}{2} \sum_{\alpha \in \Delta^{+}} \alpha$.

Next let us recall some basic facts about the biinvariant differential operators on $\mathcal{G}$. Let $U$ denote the universal enveloping algebra of $\mathcal{G}$, and $Z(U)$ be its center. Then any element $D$ in $Z(U)$ gives us a biinvariant differntial operator on $\mathcal{G}$. Given any irreducible representation $V$ of $G$, by Schur's lemma, we know that the induced action of $D$ on $V$, commuting with the action of $G$, is a multiplication by a scalar, that is

$$
D v=p_{D}(\lambda) v, v \in V,
$$

where $p_{D}$ is a polynomial. We record the following lemma ([Fe], Theorem 10.4) for reference.

Lemma 1. If $C^{W}(\mathcal{T})$ is the space of Weyl group invariant polynomials on the Lie algebra $\mathcal{T}$ and $C^{G}(\mathcal{G})$ the $A d G$ invariant polynomials on $\mathcal{G}$, then these two spaces are isomorphic and

$$
p_{D}: Z(U) \rightarrow C^{W}(\mathcal{T}) \simeq C^{G}(\mathcal{G})
$$

is an isomorphism.

In fact $p_{D}$ is a polynomial in $\lambda+\rho$. For example, if $X_{1}, \cdots, X_{n}$ is an orthonormal basis for $\mathcal{G}$, then $\mathcal{C}=X_{1}^{2}+\cdots+X_{n}^{2} \in Z(U)$ is the Casimir element. The polynomial $p_{c}(\lambda)$ is given by

$$
p_{c}(\lambda)=\|\lambda+\rho\|^{2}-\|\rho\|^{2}
$$

where the norm is induced by the Killing form.

\section{Witten's formulas}

Let $\bar{S}$ be a compact Riemann surface of genus $g>1$ and $o \in \bar{S}$ be a fixed point. Let $S=\bar{S}-D$ where $D$ is a small disc with center $o$. Let $\partial S$ be the boundary of $S$. We take an element $c \in T$, let $Z_{c}$ be the centralizer of $c$ in $G$ and

$$
\Theta_{c}=\left\{x c x^{-1} ; x \in G\right\} \simeq G / Z_{c}
$$


be the conjugacy class containing $c$. Let $\Omega^{1}(S$, ad $P)$ be the space of smooth $\mathcal{G}$-valued one forms on $S$ which vanish on $\partial S$. Define a two form on $\Omega^{1}(S$, ad $P)$ by $([\mathrm{AB}],[\mathrm{Ch} 1],[\mathrm{J}],[\mathrm{W}])$

$$
\omega(a, b)=\frac{1}{4 \pi^{2}} \int_{S}<a, b>
$$

where $a, b \in \Omega^{1}(S$, ad $P)$.

Consider the following map

$$
\begin{aligned}
& f: G^{2 g}=G \times G \cdots \times G \rightarrow G \\
& f\left(y_{1}, z_{1}, \cdots, y_{g}, z_{g}\right)=\prod_{j=1}^{g} y_{j} z_{j} y_{j}^{-1} z_{j}^{-1} .
\end{aligned}
$$

For $c=u \in Z(G)$, the center of $G$, let $\mathcal{M}_{u}=f^{-1}(u) / G$ which is the moduli space of flat principal $G$-bundles on $S$ with fixed holonomy $u \in G$ around $\partial S$. Here $G$ acts on $f^{-1}(u)$ as follows. Let $\gamma$ be the action of $G$ on $G^{2 g}$ given by

$$
\gamma(x)\left(y_{1}, z_{1}, \cdots, y_{g}, z_{g}\right)=\left(x y_{1} x^{-1}, \cdots, x z_{g} x^{-1}\right) .
$$

Then $\gamma$ induces an action on $f^{-1}(u)$. Note that $Z(G)$ acts trivially on $G^{2 g}$.

Let $\omega_{u}$ be the natural symplectic form on $\mathcal{M}_{u}$ induced by (1). From now on we will assume that $u$ is a regular value of $f$, that is $\mathcal{M}_{u}$ is a smooth compact manifold. Then the first formula we are about to prove is

Formula 1.

$$
\int_{\mathcal{M}_{u}} e^{\omega_{u}}=\# Z(G)\left[\frac{\operatorname{Vol}(G)}{(2 \pi)^{\operatorname{dim} G}}\right]^{2 g-2} \sum_{\lambda \in P_{+}} \frac{\chi_{\lambda}\left(u^{-1}\right)}{d_{\lambda}^{2 g-1}},
$$

where $\# Z(G)$ denotes the number of elements in $Z(G)$.

Witten used the notation

$$
\Lambda_{\lambda}\left(u^{-1}\right)=\frac{\chi_{\lambda}\left(u^{-1}\right)}{d_{\lambda}}
$$

More generally let us take a generic element $c \in T$, then $Z_{c}=T$ and $\Theta_{c} \simeq G / T$. Let $\mathcal{M}_{c}=f^{-1}(c) / Z_{c}$ be the moduli space of principal $G$ bundles with fixed holonomy in $\Theta_{c}$ around $\partial S$. Let $\omega_{c}$ be the natural symplectic form on $\mathcal{M}_{c}$ induced by (1). Still we assume that $c$ is a regular value of $f$, therefore $\mathcal{M}_{c}$ is a smooth compact manifold. Let $C \in \mathcal{T}$ be such that $\exp C=c$, then we have 


\section{Formula 2.}

$$
\int_{\mathcal{M}_{c}} e^{\omega_{c}}=|j(c)| \frac{\# Z(G) \operatorname{Vol}(G)^{2 g-1}}{(2 \pi)^{\operatorname{dim} \mathcal{M}_{c} \operatorname{Vol}(T)}} \sum_{\lambda \in P_{+}} \frac{\chi_{\lambda}\left(c^{-1}\right)}{d_{\lambda}^{2 g-1}},
$$

where $|j(c)|$ is the absolute value of

$$
j(c)=\prod_{\alpha \in \Delta^{+}}\left(e^{\sqrt{-1} \alpha(C) / 2}-e^{-\sqrt{-1} \alpha(C) / 2}\right) .
$$

The next formula gives us the intersection number of certain characteristic classes on $\mathcal{M}_{u}=f^{-1}(u) / G$ with $u \in Z(G)$. Note that for suitably chosen $u, \mathcal{M}_{u}$ is a smooth compact manifold of dimension $\operatorname{dim} G(2 g-2)$.

Let $f^{-1}(u) \rightarrow \mathcal{M}_{u}$ be the principal $G / Z(G)$ bundle. The $G$-equivariant cohomology of $f^{-1}(u)$ is isomorphic to the ordinary cohomology of $\mathcal{M}_{u}$. Here we take cohomology with rational coefficient.

Given any Ad-invariant homogeneous polynomial $p \in C^{W}(\mathcal{T}) \simeq$ $C^{G}(\mathcal{G}) \subset H^{*}(B G)$ of degree $2 m$. Let $2 \pi \Omega \in \Omega^{2}\left(f^{-1}(u)\right) \otimes \mathcal{G}$ be the curvature of the principal bundle $f^{-1}(u) \rightarrow \mathcal{M}_{u}$. Then $p(\sqrt{-1} \Omega) \in H^{*}\left(\mathcal{M}_{u}\right)$ is one of the generators in $H^{*}\left(\mathcal{M}_{u}\right)$. Let $\left|\Delta^{+}\right|$be the number of elements on $\Delta^{+}$, then we have

Formula 3. If $2 m<\left|\Delta^{+}\right|(2 g-2)$, then

$$
\int_{\mathcal{M}_{u}} p(\sqrt{-1} \Omega) e^{\omega_{u}}=\# Z(G)\left[\frac{\operatorname{Vol}(G)}{(2 \pi)^{\operatorname{dim} G}}\right]^{2 g-2} \sum_{\lambda \in P_{+}} \frac{\chi_{\lambda}\left(u^{-1}\right)}{d_{\lambda}^{2 g-1}} p(\lambda+\rho) .
$$

The condition on the degree of $p$ is for the convergence of the infinite sum. In fact, given any polynomial $p \in C^{W}(\mathcal{T})$, let us write

$$
A=\# Z(G)\left[\frac{\operatorname{Vol}(G)}{(2 \pi)^{\operatorname{dim} G}}\right]^{2 g-2} .
$$

Then we have the following formula:

$$
\int_{\mathcal{M}_{u}} p(\sqrt{-1} \Omega) e^{\omega_{u}}=A \lim _{c \rightarrow u} \lim _{t \rightarrow 0^{+}} \sum_{\lambda \in P_{+}} \frac{\chi_{\lambda}\left(c^{-1}\right)}{d_{\lambda}^{2 g-1}} p(\lambda+\rho) e^{-p_{c}(\lambda) t} .
$$

This formula is very similar to the one in [Liu], Lemma 6 .

When the degree of $p$ is big, the following fomula tells us that the above integral vanishes. 
Formula 4. Assume $c^{-1}=\exp H_{c}$ is such that $H_{c} \in \mathcal{T}$ does not lie in the lattice $\frac{1}{2 \pi} \Gamma$. Then for any homogegeous polynomial $p \in C^{W}(\mathcal{T})$, we have

$$
\lim _{t \rightarrow 0^{+}} \sum_{\lambda \in P_{+}} \chi_{\lambda}\left(c^{-1}\right) p(\lambda+\rho) e^{-p_{c}(\lambda) t}=0 .
$$

In particular, Formula 4 tells us that, if the degree of the polynomial $p$ in Formula 3 satifies $2 m \geq\left|\Delta^{+}\right|(2 g-2)$, and $u$ is as in Formula 4, then

$$
\int_{\mathcal{M}_{u}} p(\sqrt{-1} \Omega) e^{\omega_{u}}=0
$$

All of the above formulas can be generalized to the case when the group $G$ is not simply connected. This will be discussed in the last section of this paper.

Note that Formulas 3 and 4 give us a lot of information about the intersection numbers on $\mathcal{M}_{u}$. In particular, Formula 3 contains the information needed for the Verlinde formula. In fact, let $\hat{A}\left(T \mathcal{M}_{u}\right)$ be the $\hat{A}$-calss of $T \mathcal{M}_{u}$, then

$$
\hat{A}\left(T \mathcal{M}_{u}\right)=1+p_{1}(\sqrt{-1} \Omega)+p_{2}(\sqrt{-1} \Omega)+\cdots,
$$

where $p_{j} \in C^{W}(\mathcal{T})$ is a homogeneous polynomial of dgree $2 j$. It is easy to see that, for any integer $k$, Formulas 3 and 4 contain all the information of the integral

$$
\int_{\mathcal{M}_{u}} \hat{A}\left(T \mathcal{M}_{u}\right) e^{k \omega_{u}} .
$$

Combine with the Atiyah-Singer index formula, this gives the dimension of the nonabelian theta-functions on $\mathcal{M}_{u}$. We refer to [Sz], (see also [Mo]), for the discussion about the equivalence of this formula to the Verlinde formula for $G=S U(n)$.

\section{Heat kernel and the proof of formula 4}

Let $\triangle$ be the Laplace-Beltrami operator with respect to the biinvariant metric $\langle\cdot, \cdot>$ on $G$. One knows that $\triangle=-\mathcal{C}$. Consider, for $x, y \in G$,

$$
H(t, x, y)=\sum_{\lambda \in P_{+}} d_{\lambda} \chi_{\lambda}\left(x y^{-1}\right) e^{-p_{c}(\lambda) t} .
$$

Then $H(t, x, y)$ satisfies the following [U]

a) $\frac{\partial}{\partial t} H(t, x, y)=\triangle_{x} H(t, x, y)$,

b) $\lim _{t \rightarrow 0^{+}} \int_{G} H(t, x, y) f(y) d y=\operatorname{Vol}(G) f(x)$, 
where $d y$ is the volume element corresponding to the biinvariant metric, and $f$ is any continuous function on $G$. Therefore $\frac{1}{\operatorname{vol}(G)} H(t, x, y)$ is the standard heat kernel on $G$. Recall that for $y=u \in Z(G)$, one has

$$
d_{\lambda} \chi_{\lambda}\left(x u^{-1}\right)=\chi_{\lambda}(x) \chi_{\lambda}\left(u^{-1}\right),
$$

so we have, for $u \in Z(G)$,

$$
H(t, x, u)=\sum_{\lambda \in P_{+}} \chi_{\lambda}(x) \chi_{\lambda}\left(u^{-1}\right) e^{-p_{c}(\lambda) t} .
$$

Before the start of the proofs of the formulas in $\S 2$, we first compute the integral of the pull-back of the heat kernel on $G$ by $f$ :

$$
\int_{G^{2 g}} H(t, f(h), x) d \mathrm{vol},
$$

where $h=\left(y_{1}, z_{1}, \cdots, y_{g}, z_{g}\right) \in G^{2 g}$ and $d$ vol is the product volume element on $G^{2 g}$. Obviously we only need to compute the $2 g$-iterated integrals like

$$
\int_{G} \cdots \int_{G} \chi_{\lambda}\left(x^{-1} \prod_{j=1}^{g} y_{j} z_{j} y_{j}^{-1} z_{j}^{-1}\right) \prod_{j=1}^{g} d y_{j} d z_{j}
$$

where $d y_{j}, d z_{j}$ denote the volume element on $G$ induced by the Killing form.

Recall the standard formulas in the representation theory of compact Lie groups which follow from the orthogonal relations ([BD], pp 84):

$$
\int_{G} \chi_{\lambda}\left(w y z y^{-1} z^{-1}\right) d z=\frac{\operatorname{Vol}(\mathrm{G})}{d_{\lambda}} \chi_{\lambda}(w y) \chi_{\lambda}\left(y^{-1}\right),
$$

and

$$
\int_{G} \chi_{\lambda}(w y) \chi_{\lambda}\left(y^{-1}\right) d y=\frac{\operatorname{Vol}(\mathrm{G})}{d_{\lambda}} \chi_{\lambda}(w)
$$

from which we get

$$
\int_{G^{2 g}} \chi_{\lambda}\left(x^{-1} \prod_{j=1}^{g} y_{j} z_{j} y_{j}^{-1} z_{j}^{-1}\right) \prod_{j=1}^{g} d y_{j} d z_{j}=\frac{\operatorname{Vol}(G)^{2 g}}{d_{\lambda}^{2 g-1}} \chi_{\lambda}\left(x^{-1}\right) .
$$

We summarize this as a lemma.

Lemma 2. The following formula holds

$$
\int_{G^{2 g}} H(t, f(h), x) d \mathrm{vol}=\operatorname{Vol}(G)^{2 g} \sum_{\lambda \in P_{+}} \frac{\chi_{\lambda}\left(x^{-1}\right)}{d_{\lambda}^{2 g-1}} e^{-p_{c}(\lambda) t} .
$$


Next we want to prove Formula 4, the vanishing theorem. This is just a simple application of the Poisson summation formula. Let $c$ be such that $c^{-1}=\exp H_{c}$ with $H_{c}$ not in the lattice $\frac{1}{2 \pi} \Gamma=\sum_{j=1}^{l} Z H_{\alpha_{j}}^{v}$. Consider the series

$$
Z\left(t, H_{c}\right)=\sum_{\lambda \in P_{+}} p(\lambda+\rho) \chi_{\lambda}\left(c^{-1}\right) e^{-p_{c}(\lambda) t}
$$

where $p \in C^{W}(\mathcal{T})$ is a homogeneous polynomial.

By using the Weyl character formula we can rewrite $Z\left(t, H_{c}\right)$ as

$$
Z\left(t, H_{c}\right)=\frac{e^{\|\rho\|^{2} t}}{j\left(c^{-1}\right)} \sum_{\lambda \in P_{+}} \sum_{w \in W} \varepsilon(w) p(\lambda+\rho) e^{-\|\lambda+\rho\|^{2} t} e^{\sqrt{-1} w(\lambda+\rho)\left(H_{c}\right)} .
$$

Let $\# W$ denote the number of elements in the Weyl group $W$. We get

$$
\begin{aligned}
Z\left(t, H_{c}\right) & =\frac{e^{\|\rho\|^{2} t}}{\# W j\left(c^{-1}\right)} \sum_{\lambda \in I} \sum_{w \in W} \varepsilon(w) p(\lambda+\rho) e^{-\|\lambda+\rho\|^{2} t} e^{\sqrt{-1} w(\lambda+\rho)\left(H_{c}\right)} \\
& =\frac{e^{\|\rho\|^{2} t}}{\# W j\left(c^{-1}\right)} \sum_{\lambda \in I} \sum_{w \in W} \varepsilon(w) p(\lambda) e^{-\|\lambda\|^{2} t} e^{\sqrt{-1} \lambda\left(w\left(H_{c}\right)\right)},
\end{aligned}
$$

where $w$ acts on $H_{c} \in \mathcal{T}$ by adjoint action through the identification $W \simeq N(T) / T$. Here $N(T)$ denotes the normalizer of $T$.

Consider the Fourier transform $\hat{F}(H)$ of

$$
F(\lambda)=P(\lambda) e^{-\|\lambda\|^{2} t} e^{\sqrt{-1} \lambda\left(w\left(H_{c}\right)\right)} .
$$

Here by definition

$$
\hat{F}(H)=\frac{1}{(2 \pi)^{l / 2}} \int_{\mathcal{T}^{*}} F(\lambda) e^{-\sqrt{-1} \lambda(H)} d \lambda .
$$

It is easy to carry out this integration which gives

$$
\hat{F}(H)=R\left(t, H-w\left(H_{c}\right)\right) e^{-\frac{1}{4 t}\left\|H-w\left(H_{c}\right)\right\|^{2}},
$$

where $R\left(t, H-w\left(H_{c}\right)\right)$ is a polynomial in $\left(H-w\left(H_{c}\right)\right)$ and $\frac{1}{\sqrt{t}}$. By applying the Poisson summation formula, we get

$$
Z\left(t, H_{c}\right)=\frac{e^{\|\rho\|^{2} t}}{\# W j\left(c^{-1}\right)} \sum_{H \in \frac{1}{2 \pi} \Gamma} \sum_{w \in W} \varepsilon(w) R\left(t, H-w\left(H_{c}\right)\right) e^{-\frac{1}{4 t}\left\|H-w\left(H_{c}\right)\right\|^{2}} .
$$

By our assumption, $H_{c}$ does not belong to the lattice $\frac{1}{2 \pi} \Gamma$, so for $H \in$ $\frac{1}{2 \pi} \Gamma, H-w\left(H_{c}\right)$ is never zero. Therefore when $t \rightarrow 0^{+}$, the left hand side exponentially goes to zero. This proves Formula 4. 


\section{The proofs of formulas 1,2 and 3}

Now we start to prove Formula 1. We will assume that $u \in Z(G)$ is a regular value of $f$, therefore $\mathcal{M}_{u}$ is a smooth compact manifold.

From Sect. 1 we know that, when $t \rightarrow 0^{+}$,

$$
\int_{G^{2 g}} H(t, f(h), u) d \mathrm{vol}=\int_{f^{-1}\left(B_{\delta}\right)} H(t, f(h), u) d \mathrm{vol}+O\left(e^{-\delta / t}\right),
$$

where $B_{\delta} \subset G$ is a ball of radius $\delta$ around $u$.

The following local calculation is basically due to [Fo]. Given a point $a \in f^{-1}(u)$, we can choose local coordinate around $a$ by using

$$
(a, b) \in f^{-1}(u) \times N_{a} \simeq f^{-1}\left(B_{\delta}\right),
$$

where $N_{a}$ is the fiber at $a$ of the normal bundle in $G^{2 g}$ to $f^{-1}(u)$. By using exponential map, we will identify $b$ to the tangent vector in $N_{a}$. Then the volume element $d$ vol at $(a, b)$ becomes

$$
d \mathrm{vol}=J(a, b) d \operatorname{vol}_{a} d \operatorname{vol}_{b},
$$

where $d \mathrm{vol}_{a}$ and $d \mathrm{vol}_{b}$ are respectively the induced Riemannian volume elements of $f^{-1}(u)$ and $N$ at $(a, b)$, and $J(a, b)$ with $J(a, 0)=1$ is the Jacobian of the coordinate change.

In this coordinate $h=(a, b)$, and

$$
\|f(h)-u\|^{2}=\|[d f(a)](b)\|^{2}+O\left(\|b\|^{3}\right),
$$

from which we get

$$
H(t, f(h), u)=\frac{\operatorname{Vol}(G)}{(4 \pi t)^{\operatorname{dim} G / 2}} e^{-\left(\|[d f(a)](b)\|^{2}+O\left(\|b\|^{3}\right)\right) / 4 t}(1+O(t)) .
$$

So the integral has the asymptotics

$$
\begin{aligned}
& \int_{a \in f^{-1}(u)} d \operatorname{vol}_{a} \\
& \quad\left[\int_{N_{a}} \frac{\operatorname{Vol}(G)}{(4 \pi t)^{\operatorname{dim} G / 2}} e^{-\left(\|[d f(a)](b)\|^{2}+O\left(\|b\|^{3}\right)\right) / 4 t}(1+O(t)) J(a, b) d \operatorname{vol}_{b}\right] .
\end{aligned}
$$

By changing variable $b \rightarrow \sqrt{t} b$, carrying out the standard Gaussian integral and letting $t$ go to zero, we get

$$
\begin{aligned}
& \int_{N_{a}} \frac{\operatorname{Vol}(G)}{(4 \pi t)^{\operatorname{dim} G / 2}} e^{-\left(\|[d f(a)](b)\|^{2} O\left(\|b\|^{3}\right)\right) / 4 t}(1+O(t)) J(a, b) d \operatorname{vol}_{b} \\
& =\left.\operatorname{Vol}(G) \operatorname{det}^{-\frac{1}{2}} d f^{*}(a) d f(a)\right|_{N_{a}} .
\end{aligned}
$$


Here let $\left\{t_{1}, \cdots, t_{n}\right\}$ be a basis for the orthogonal complement of the kernel of $d f(a): \mathcal{G}^{2 g} \rightarrow \mathcal{G},[\operatorname{Ker} d f(a)]^{\perp} \simeq \mathcal{G}$, then

$$
\left.\operatorname{det}^{-\frac{1}{2}} d f^{*}(a) d f(a)\right|_{N_{a}}=\frac{\left|t_{1} \wedge \cdots \wedge t_{n}\right|}{\left|[d f(a)]\left(t_{1}\right) \wedge \cdots \wedge[d f(a)]\left(t_{n}\right)\right|},
$$

where $\left|t_{1} \wedge \cdots \wedge t_{n}\right|$ denotes absolute value of the determinant $\left|<t_{i}, t_{j}>\right|$ and $\mathcal{G}^{2 g}$ is the direct sum of $2 g$ copies of the Lie algebra $\mathcal{G}$.

Therefore we have

$$
\begin{aligned}
\lim _{t \rightarrow 0^{+}} & \int_{G^{2 g}} H(t, f(h), u) d \mathrm{vol}= \\
& \left.\operatorname{Vol}(G) \int_{a \in f^{-1}(u)} \operatorname{det}^{-\frac{1}{2}} d f^{*}(a) d f(a)\right|_{N_{a}} d \operatorname{vol}_{u} .
\end{aligned}
$$

On the other hand, since the volume of the orbit of $\gamma$, the induced conjugate action, through $a \in f^{-1}(u)$ is given by

$$
\frac{\operatorname{Vol}(G)}{\# Z(G)} \operatorname{det}^{\frac{1}{2}}\left[d \gamma^{*}(a) d \gamma(a)\right]
$$

where, for a basis $\left\{s_{1}, \cdots, s_{n}\right\}$ of $\mathcal{G}$,

$$
\operatorname{det}^{\frac{1}{2}}\left[d \gamma^{*}(a) d \gamma(a)\right]=\frac{\left|[d \gamma(a)]\left(s_{1}\right) \wedge \cdots \wedge[d \gamma(a)]\left(s_{n}\right)\right|}{\left|s_{1} \wedge \cdots \wedge s_{n}\right|} .
$$

Note that the action of $Z(G)$ on $f^{-1}(u)$ is trivial. Now it is easy to reduce the integral over $f^{-1}(u)$ to $\mathcal{M}_{u}=f^{-1}(u) / G$. We get

$$
\left.\int_{a \in f^{-1}(u)} \operatorname{det}^{-\frac{1}{2}}\left[d f^{*}(a) d f(a)\right]\right|_{N_{a}} d \operatorname{vol}_{a}=\frac{\operatorname{Vol}(G)^{2}}{\# Z(G)} \int_{\mathcal{M}_{u}} d \nu_{u},
$$

where $d \nu_{u}$ is the measure on $\mathcal{M}_{u}$ defined by the following property: for any basis $\left\{r_{1}, \cdots, r_{N}\right\}$ of $T_{a} \mathcal{M}$,

$$
\begin{aligned}
& d \nu_{u}\left(r_{1} \wedge \cdots \wedge r_{N}\right)= \\
& \frac{\left|r_{1} \wedge \cdots \wedge r_{N} \wedge[d \gamma(a)]\left(s_{1}\right) \wedge \cdots \wedge[d \gamma(a)]\left(s_{n}\right) \wedge t_{1} \wedge \cdots \wedge t_{n}\right|}{\left|s_{1} \wedge \cdots \wedge s_{n}\right|\left|[d f(a)]\left(t_{1}\right) \wedge \cdots \wedge[d f(a)]\left(t_{n}\right)\right|} .
\end{aligned}
$$

We summarize the above calculations as

Lemma 3. We have the following integral formula

$$
\lim _{t \rightarrow 0^{+}} \int_{G^{2 g}} H(t, f(h), u) d \operatorname{vol}=\frac{\operatorname{Vol}(G)^{2}}{\# Z(G)} \int_{\mathcal{M}_{u}} d \nu_{u},
$$

where $d \nu_{u}$ is defined as above. 
Now let us understand $d \nu_{u}$. At $a \in f^{-1}(u)$, we have the following chain complex,

$$
\mathcal{C}_{a}: 0 \rightarrow \mathcal{G} \stackrel{d \gamma}{\rightarrow} \mathcal{G}^{2 g} \stackrel{d f}{\rightarrow} \mathcal{G} \rightarrow 0 .
$$

Here we have identified the tangent bundles of the $G$ 's with their Lie algebras by using left translations. Note that $\mathcal{C}_{a}$ is the deformation complex of the flat principal $G$-bundle on $\bar{S}$ with fixed holonomy $u \in G$ around $o$. On each term in $\mathcal{C}_{a}$, there is the natural measure induced by $\langle\cdot, \cdot\rangle$. Note that, $H^{0}\left(\mathcal{C}_{a}\right)=H^{2}\left(\mathcal{C}_{a}\right)=0$ and $H^{1}\left(\mathcal{C}_{a}\right) \simeq T_{a} \mathcal{M}_{u}$. Let us denote by $\tau\left(\mathcal{C}_{a}\right)$ the torsion of $\mathcal{C}_{a}$. We consider $\tau\left(\mathcal{C}_{a}\right)$ as a norm on $\operatorname{det} H^{1}\left(\mathcal{C}_{a}\right) \simeq \operatorname{det} T_{a} \mathcal{M}_{u}$. On the other hand, from its definition $d \nu_{u}$ can also be viewed as a norm on $T_{a} \mathcal{M}_{u}$. The following combinatorics lemma is due to Witten [W] and was first applied in this situation in [Fo].

Lemma 4. Let $N=\operatorname{dim} \mathcal{M}_{u}$, then

$$
d \nu_{u}=\tau\left(\mathcal{C}_{a}\right)=(2 \pi)^{N} \frac{\omega_{u}^{N}}{N !}
$$

Remark. Zhang outlined to me a very simple proof of Lemma 4 . In fact $\tau\left(\mathcal{C}_{a}\right)$ is the Reidemeister metric on $\operatorname{det} H^{1}\left(\mathcal{C}_{a}\right)$ ([BZ], [Fa]) which is equal to the $L^{2}$-metric on any compact even dimensional manifold. In our case, the $L^{2}$-metric on $\operatorname{det} H^{1}\left(\mathcal{C}_{a}\right) \simeq \operatorname{det} T_{a} \mathcal{M}_{u}$ is the same as the symplectic volume. Zhang used Ray-Singer metric, but by the Ray-Singer-CheegerMuller theorem, we know that this is the same as the Reidemeister metric. In fact both Lemma 4 nd Lemma $4 \mathrm{c}$ below are simple generalizations of the duality result in [Mi].

For completeness, here we sketch the proof of Witten. The first equality is basically the definition of the torsion for the complex $\mathcal{C}_{a}[\mathrm{Fo}]$. For the second equality, we consider the standard cell decomposition of $\bar{S}$ given by the $4 g$-sided polygons which gives the well-known description of the fundemental group of $\bar{S}$. It has one 0 -cell, one 2 -cell and $2 g 1$-cells. The complex $\mathcal{C}_{a}$ is precisely the "lattice model" of the gauge theory of a flat principal $G$-bundle on $\bar{S}$ ([Fo], pp 41). Note that $\tau\left(\mathcal{C}_{a}\right)$ is invariant under subdivision of the cell decomposition. The dual cell decomposition gives us a dual complex $\mathcal{C}_{a}^{\prime}$ to $\mathcal{C}_{a}$. Poincaré duality induces a natural skewsymmetric pairing on the complex $\mathcal{D}_{a}=\mathcal{C}_{a} \oplus \mathcal{C}_{a}^{\prime}$ which is compatible with both the differentials and the natural measures on each term in $\mathcal{D}_{a}$. Therefore, since $H^{1}\left(\mathcal{C}_{a}\right) \simeq T_{a} \mathcal{M}_{u}$, it induces a skew-symmetric pairing

$$
T_{a} \mathcal{M}_{u} \times T_{a} \mathcal{M}_{u} \rightarrow R
$$


which is precisely the natural symplectic form $4 \pi^{2} \omega_{u}$ on $\mathcal{M}_{u}$ ([W], (4.16) to $(4.28))$. So one has $([\mathrm{W}],(4.28))$

$$
\sqrt{\tau\left(\mathcal{D}_{a}\right)}=(2 \pi)^{N} \frac{\omega_{u}^{N}}{N !} .
$$

Since $\tau\left(\mathcal{D}_{a}\right)=\tau\left(\mathcal{C}_{a}\right) \tau\left(\mathcal{C}_{a}^{\prime}\right)=\tau\left(\mathcal{C}_{a}\right)^{2}$, we get

$$
\tau\left(\mathcal{C}_{a}\right)=(2 \pi)^{N} \frac{\omega_{u}^{N}}{N !} .
$$

We thus have obtained the following equality, which is exactly Formula 1,

$$
\int_{\mathcal{M}_{u}} e^{\omega_{u}}=\# Z(G)\left[\frac{\operatorname{Vol} G}{(2 \pi)^{\operatorname{dim} G}}\right]^{2 g-2} \sum_{\lambda \in P_{+}} \frac{\chi_{\lambda}\left(u^{-1}\right)}{d_{\lambda}^{2 g-1}} .
$$

Note that here we have assumed $G$ is simply connected and $u \in Z(G)$ is a regular point of $f$.

Now let us prove Formula 2. Since $c$ is a generic element, the centralizer of $c, Z_{c}=T$. The integral we will consider is

$$
\int_{G^{2 g}} H(t, f(h), c) d \mathrm{vol}
$$

Lemma 2 gives us its value as the infinite sum, we now consider its localization to $f^{-1}(c)$ when $t$ goes to zero.

Similar to the proof of Formula 1, we can easily get

$$
\begin{aligned}
\lim _{t \rightarrow 0^{+}} & \int_{G^{2 g}} H(t, f(h), c) d \mathrm{vol}= \\
& \left.\operatorname{Vol}(G) \int_{a \in f^{-1}(c)} \operatorname{det}^{-\frac{1}{2}} d f^{*}(a) d f(a)\right|_{N_{a}} d \operatorname{vol}_{a}
\end{aligned}
$$

where $d \mathrm{vol}_{a}$, is the induced Riemannian volume element on $f^{-1}(c)$.

Note that $f^{-1}(c)$ is only invariant under the action of $Z_{c}=T$. Let $\gamma_{c}$ be the restriction of the conjugate action $\gamma$ to $Z_{c}$. Since the volume of the orbit of $\gamma_{c}$ through $a$ is

$$
\frac{\operatorname{Vol}(T)}{\# Z(G)} \operatorname{det}^{\frac{1}{2}}\left[d \gamma_{c}^{*}(a) d \gamma_{c}(a)\right]
$$

where, for a basis $\left\{s_{1}, \cdots s_{l}\right\}$ of $Z_{c}=T$,

$$
\operatorname{det}^{\frac{1}{2}}\left[d \gamma_{c}^{*}(a) d \gamma_{c}(a)\right]=\frac{\left|\left[d \gamma_{c}(a)\right]\left(s_{1}\right) \wedge \cdots \wedge\left[d \gamma_{c}(a)\right]\left(s_{l}\right)\right|}{\left|s_{1} \wedge \cdots \wedge s_{l}\right|}
$$


In the same way we can reduce the integral on the right hand side to

$$
\left.\int_{a \in f^{-1}(c)} \operatorname{det}^{-\frac{1}{2}} d f^{*}(a) d f(a)\right|_{N_{a}} d \operatorname{vol}_{a}=\operatorname{Vol}(G) \frac{\operatorname{Vol}(T)}{\# Z(G)} \int_{\mathcal{M}_{c}} d \nu_{c}
$$

where $d \nu_{c}$ is a measure on $\mathcal{M}_{c}$, such that for a basis $\left\{r_{1}, \cdots, r_{K}\right\}$ of $T_{a} \mathcal{M}_{c}$,

$$
\begin{gathered}
d \nu_{c}\left(r_{1} \wedge \cdots \wedge r_{K}\right) \\
=\frac{\left|r_{1} \wedge \cdots \wedge r_{K} \wedge\left[d \gamma_{c}(a)\right]\left(s_{1}\right) \wedge \cdots \wedge\left[d \gamma_{c}(a)\right]\left(s_{l}\right) \wedge t_{1} \wedge \cdots \wedge t_{n}\right|}{\left|s_{1} \wedge \cdots \wedge s_{l}\right|\left|[d f(a)]\left(t_{1}\right) \wedge \cdots \wedge[d f(a)]\left(t_{n}\right)\right|} .
\end{gathered}
$$

Here $\left\{t_{1}, \cdots, t_{n}\right\}$ still denote a basis of the orthogonal complement to $\operatorname{Ker} d f(a)$. From the above definition, we see that $d \nu_{c}$ can be considered as a norm on $\operatorname{det} T_{a} \mathcal{M}_{c}$. Similar to Lemma 3, we can get

Lemma 5. We have

$$
\lim _{t \rightarrow 0^{+}} \int_{G^{2 g}} H(t, f(h), c) d \operatorname{vol}=\operatorname{Vol}(G) \frac{\operatorname{Vol}(T)}{\# Z(G)} \int_{\mathcal{M}_{c}} d \nu_{c} .
$$

Now let us understand $d \nu_{c}$ which will be related to the symplectic volume on $\mathcal{M}_{c}$. Let $\mathcal{Z}_{c}$ denote the Lie algebra of $Z_{c}$. We consider the following chain complex at $a \in f^{-1}(c)$,

$$
\mathcal{C}_{a}^{c}: 0 \rightarrow \mathcal{Z}_{c} \stackrel{d \gamma_{c}}{\rightarrow} \mathcal{G}^{2 g} \stackrel{d f}{\rightarrow} \mathcal{G} \rightarrow 0 .
$$

This complex is the "lattice model" associated to the cell decomposition of the pair $(S, \partial S)$. Here we still identify the tangent bundle of $G$ with $\mathcal{G}$ by using left translations. Note that by definition [Fo] we have similarly $\tau\left(\mathcal{C}_{a}^{c}\right)=d \nu_{c}$.

The complex associated to the Poincaré dual complex of $\mathcal{C}_{a}^{c}$ is given explicitly by the following

$$
\mathcal{C}_{a}^{c^{\prime}}: 0 \rightarrow \mathcal{G} \stackrel{d \gamma_{0}}{\rightarrow} \mathcal{G}^{2 g} \oplus T_{c} \Theta_{c} \stackrel{d f_{0}}{\rightarrow} \mathcal{G} \rightarrow 0,
$$

where $T_{c} \Theta_{c}$ is the fiber at $c$ of the tangent bundle of the orbit $\Theta_{c} \simeq G / T$ of $c$ in $G$ under the conjugate action $\gamma$. It is identified with $\mathcal{Z}_{c}^{\perp}$, the orthogonal complement of $\mathcal{Z}_{c}$ in $\mathcal{G}$, through left translation by $c^{-1}$. The $\gamma_{0}$, an extension of $\gamma$, is the conjugate action of $G$ on $G \times \Theta_{c}$ given by

$$
\gamma_{0}(x)\left(y_{1}, \cdots, z_{g} ; y\right)=\left(x y_{1} x^{-1}, \cdots, x z_{g} x^{-1} ; x y x^{-1}\right),
$$

and the $f_{0}$ is the map from $G^{2 g} \times \Theta_{c}$ to $G$ given by

$$
f_{0}\left(\left(y_{1}, \cdots, z_{g} ; y\right)\right)=y^{-1} \prod_{j=1}^{g} y_{j} z_{j} y_{j}^{-1} z_{j}^{-1} \text {. }
$$


It is easy to see that $\mathcal{M}_{c} \simeq f_{0}^{-1}(e) / G$.

Note that $\mathcal{C}_{a}^{c \prime}$ is the "lattice model" complex associated to the cell decomposition of $S$ which gives the standard description of the fundemental group of $S$. It has one 0 -cell, one 2-cell and $2 g+11$-cells. One of the 1-cells is the boundary circle $\partial S$. We need to associate $\Theta_{c}$ to $\partial S$ (cf. [W], [Fo]).

We have the following exact sequence

$$
0 \rightarrow \mathcal{C}_{a}^{c} \rightarrow \mathcal{C}_{a}^{c \prime} \rightarrow K_{c} \rightarrow 0
$$

where $K_{c}$ is the complex

$$
0 \rightarrow \mathcal{Z}_{c}^{\perp} \stackrel{d \gamma_{0}}{\rightarrow} T_{c} \Theta_{c} \rightarrow 0 \rightarrow 0 .
$$

In fact $K_{c}$ is precisely the chain complex associated to the boundary circle $\partial S[\mathrm{~W}]$. Note that our complexes are dual to those of Witten's in [W], and the same as those in $[\mathrm{Fo}]$.

As in the derivation of the Weyl integral formula ([BT], pp 162), we easily find that the torsion of $K_{c}$ is [W]

$$
\tau\left(K_{c}\right)=\left|\operatorname{det}\left(\operatorname{Ad}\left(c^{-1}\right)-I\right)\right|=|j(c)|^{2} .
$$

So we get

$$
\tau\left(\mathcal{C}_{a}^{c \prime}\right)=\tau\left(\mathcal{C}_{a}^{c}\right) \tau\left(K_{c}\right)=\tau\left(\mathcal{C}_{a}^{c}\right)|j(c)|^{2}
$$

Note that

$$
H^{0}\left(\mathcal{C}_{a}^{c}\right)=H^{2}\left(\mathcal{C}_{a}^{c}\right)=H^{0}\left(\mathcal{C}_{a}^{c \prime}\right)=H^{2}\left(\mathcal{C}_{a}^{c \prime}\right)=0
$$

and

$$
H^{1}\left(\mathcal{C}_{a}^{c}\right) \simeq H^{1}\left(\mathcal{C}_{a}^{c \prime}\right) \simeq T_{a} \mathcal{M}_{c}
$$

We consider the complex $D_{c}=\mathcal{C}_{a}^{c} \oplus \mathcal{C}_{a}^{c \prime}$. The Poincaré duality induces a natural skew-symmetric pairing on $D_{c}$ which is compatible with both the differentials and the natural measures of $D_{c}$. Therefore it induces the symplectic structure on $\mathcal{M}_{c}$. Following Witten's argument ([W], (4.105)), in the same way as in the proof of Lemma 4 , we get

$$
\sqrt{\tau\left(D_{c}\right)}=(2 \pi)^{K} \frac{\omega_{c}^{K}}{K !}=\tau\left(\mathcal{C}_{a}^{c}\right)|j(c)|
$$

where $K$ denotes the dimension of $\mathcal{M}_{c}$. Here we have used the equality $\tau\left(D_{c}\right)=\tau\left(\mathcal{C}_{a}^{c}\right) \tau\left(\mathcal{C}_{a}^{c \prime}\right)$. Let us summarize the above discussions as a lemma.

Lemma 4c. We have the following equalities:

$$
d \nu_{c}|j(c)|=\tau\left(\mathcal{C}_{a}^{c}\right)|j(c)|=(2 \pi)^{K} \frac{\omega_{c}^{K}}{K !} .
$$


Remark. As Zhang explained to me, his simple proof of Lemma 4 applies to this case, and $\mathcal{C}_{a}^{c}, \mathcal{C}_{a}^{c \prime}$ correspond to the complexes with different boundary, absolute and relative, conditions. The term $\tau\left(K_{c}\right)$ is precisely the Reidemeister metric of the boundary ([Lu], Theorem 5.9). See also [Mi], Theorem 2 and the discussion in [RS], Sect. 3.

By putting this together with the formula in Lemma 2, we get

$$
\begin{aligned}
\int_{\mathcal{M}_{c}} e^{\omega_{c}} & =\frac{1}{(2 \pi)^{\operatorname{dim} \mathcal{M}_{c}}} \int_{\mathcal{M}_{c}} \tau\left(\mathcal{C}_{a}^{c}\right)|j(c)| \\
& =|j(c)| \frac{\# Z(G) \operatorname{Vol}(G)^{2 g-1}}{(2 \pi)^{\operatorname{dim} \mathcal{M}_{c} \operatorname{Vol}(T)}} \sum_{\lambda \in P_{+}} \frac{\chi_{\lambda}\left(c^{-1}\right)}{d_{\lambda}^{2 g-1}},
\end{aligned}
$$

which is precisely Formula 2.

Now let us compare Formula 1 and Formula 2. Let $C, H_{u}^{0} \in \mathcal{T}$ be such that $u \exp C=c$ and $u=\exp H_{u}^{0}$, we assume $C, H_{u}^{0}$ lie in the closure of the fixed Weyl chamber $\mathcal{C} \subset \mathcal{T}$. When $c$ is very near $u$ in $T=Z_{c}$, one knows that $f^{-1}(c)$ is diffeomorphic to $f^{-1}(u)$. Therefore $\mathcal{M}_{c}$ is a fiber bundle over $\mathcal{M}_{u}$ with fiber $G / T \simeq G / Z_{c}$. Let us write this as

$$
\pi: \mathcal{M}_{c} \rightarrow \mathcal{M}_{u} .
$$

Then one has the standard relation of symplectic forms $\omega_{c}=\pi^{*} \omega_{u}+\nu_{c}$, where $\nu_{c}$ is a two form on $\mathcal{M}_{c}$ which, when restricted to the fiber $G / Z_{c} \simeq$ $G / T$, is the standard symplectic form. In fact, a simple application of the local model theorem for symplectic manifold to the fibration $f^{-1}(u) \rightarrow \mathcal{M}_{u}$ gives us ([Ch], [Ch1], [D], [J])

$$
\nu_{c}=<C, \Omega>+\theta_{c},
$$

where, for $X, Y \in \mathcal{G}, \theta_{c}$ is given by ([BGV], $\S 7.5$ )

$$
\theta_{c}(X, Y)=-\frac{1}{2 \pi}<C,[X, Y]>\text {. }
$$

For the geometric meaning of $\nu_{c}$ in terms of loop group, see [Ch].

This gives us

$$
\int_{\mathcal{M}_{c}} e^{\omega_{c}}=\int_{\mathcal{M}_{u}} e^{\omega_{u}} \pi_{*} e^{\nu_{c}}
$$

where $\pi_{*}$ is the integration along a generic fiber of $\pi$. By comparing Formula 1 and Formula 2, it is easy to see that one must have

Lemma 6.

$$
\lim _{c \rightarrow u} \frac{\pi_{*} e^{\nu_{c}}}{j(c)}= \pm \frac{\operatorname{Vol}(G / T)}{(2 \pi)^{\operatorname{dim}(G / T)}}
$$


Here $\operatorname{Vol}(G / T)=\operatorname{Vol}(G) / \operatorname{Vol}(T)$ is the Riemannian volume of $G / T$. The sign \pm will be fixed later.

Let us briefly explain the geometric reason for Lemma 6 . First we know that the symplectic volume of $G / Z_{c} \simeq G / T$ with symplectic form $\theta_{c}$ is ([BGV], Lemma 7.32)

$$
\int_{G / Z_{c}} e^{\theta_{c}}=\operatorname{Vol}(G / T) \prod_{\alpha \in \Delta^{+}} \frac{\sqrt{-1} \alpha(C)}{2 \pi} .
$$

It is easy to see that the leading term of the integral $\pi_{*} e^{\nu_{c}}$ is given by the symplectic volume of the fiber, that is, we have

$$
\pi_{*} e^{\nu_{c}}=\operatorname{Vol}(G / T) \prod_{\alpha \in \Delta^{+}} \frac{\sqrt{-1} \alpha(C)}{2 \pi}+\cdots,
$$

where the $\cdots$ are those terms involving higher order terms in $\alpha(C)$. From Formula 2, we get the following identity:

$$
\begin{aligned}
& \operatorname{Vol}(G / T) \prod_{\alpha \in \Delta^{+}} \frac{\sqrt{-1} \alpha(C)}{2 \pi} \int_{\mathcal{M}_{u}} e^{\omega_{u}}+\cdots \\
& =|j(c)| \frac{\# Z(G) \operatorname{Vol}(G)^{2 g-1}}{(2 \pi)^{\operatorname{dim} \mathcal{M}_{c} \operatorname{Vol}(T)}} \sum_{\lambda \in P_{+}} \frac{\chi_{\lambda}\left(c^{-1}\right)}{d_{\lambda}^{2 g-1}} .
\end{aligned}
$$

Let $\pi(c)=\prod_{\alpha \in \Delta^{+}} \sqrt{-1} \alpha(C)$. Since $u \in Z(G)$, we know that, when $c$ goes to $u$, each $\alpha(C)$ should go to zero which implies that

$$
\lim _{c \rightarrow u} \frac{\pi(c)}{j(c)}= \pm 1
$$

which is equivalent to Lemma 6.

Now we are ready to prove Formula 3 . We will actually show that in fact Formula 3 follows from Formulas 1 and 2 in the same way as the above discussion. First let $2 \pi \Omega$ be the curvature of the principal bundle $\pi: f^{-1}(u) \rightarrow \mathcal{M}_{u}$. The following formula which was first shown to me by Chang [Ch] can be viewed as a family version of the Duistermaat-Heckman integral formula, or more generally a family version of the equivariant localization formula ([BGV], Theorem 7.33).

$$
\pi_{*} e^{\nu_{c}}=\frac{\sum_{w \in W} \varepsilon(w) e^{<w C, \Omega>}}{\prod_{\alpha \in \Delta^{+}}(-\sqrt{-1} \alpha(\Omega))} .
$$

Here the Weyl group $W$ acts on $C \in \mathcal{T}$ by the the adjoint action, through the identification $N(T) / T \simeq W$. A similar formula is derived in [KS], Proposition 5.3. 
Let $\left\{H_{1}, \cdots, H_{l}\right\}$ be an orthonormal basis of $\mathcal{T}$, write $C=x_{1} H_{1}+\cdots+$ $x_{l} H_{l} \in \mathcal{T}$. We use $\left(x_{1}, \cdots, x_{l}\right)$ as the coordinate of $C \in \mathcal{T}$. Given a $W$ invariant homogeneous polynomial on $\mathcal{T}, p\left(x_{1}, \cdots, x_{l}\right) \in C^{W}(\mathcal{T}) \simeq C^{G}(\mathcal{G})$ of degree $2 m$. Let the differential operator $p\left(\frac{\partial}{\partial x_{1}}, \cdots, \frac{\partial}{\partial x_{l}}\right)$ act on both sides of Formula 2 with respect to $C \in \mathcal{T}$. Note that

$$
p\left(\frac{\partial}{\partial x_{1}}, \cdots, \frac{\partial}{\partial x_{l}}\right) \pi_{*} e^{\nu_{c}}=p(\Omega) \pi_{*} e^{\nu_{c}},
$$

which follows from the above formula (3) (or [V], Proposition 10). On the other hand, from the Weyl character formula, we have

$$
j(c) \chi_{\lambda}\left(c^{-1}\right)=\sum_{w \in W} \varepsilon(w) e^{-\sqrt{-1} w(\lambda+\rho)(C)},
$$

which gives

$$
p\left(\frac{\partial}{\partial x_{1}}, \cdots, \frac{\partial}{\partial x_{l}}\right)\left[j(c) \chi_{\lambda}\left(c^{-1}\right)\right]=(-1)^{m} p(\lambda+\rho)\left[j(c) \chi_{\lambda}\left(c^{-1}\right)\right] .
$$

Now let $c \rightarrow u$ again, from Formula 2 and Lemma 6 we get

$$
\int_{\mathcal{M}_{u}} e^{\omega_{u}} p(\Omega)= \pm(-1)^{m} \# Z(G) \frac{\operatorname{Vol}(G)^{2 g-2}}{(2 \pi)^{\operatorname{dim} \mathcal{M}_{u}}} \sum_{\lambda \in P_{+}} \frac{\chi_{\lambda}\left(u^{-1}\right)}{d_{\lambda}^{2 g-1}} p(\lambda+\rho) .
$$

The overall sign \pm is fixed by taking $p=1$ and noting that, as the limits of the heat kernel, both sides should be positive. This proves Formula 3 .

In the above discussion we did not pay attention to the convergence. But it is easy to see that our proof actually gives the following formula:

$$
\int_{\mathcal{M}_{u}} e^{\omega_{u}} p(\sqrt{-1} \Omega)=A \lim _{c \rightarrow u} \lim _{t \rightarrow 0^{+}} \sum_{\lambda \in P_{+}} \frac{\chi_{\lambda}\left(c^{-1}\right)}{d_{\lambda}^{2 g-1}} p(\lambda+\rho) e^{-p_{c}(\lambda) t},
$$

where

$$
A=\# Z(G) \frac{\operatorname{Vol}(G)^{2 g-2}}{(2 \pi)^{\operatorname{dim} \mathcal{M}_{u}}}
$$

as defined in $\S 2$.

\section{Nonsimply connected groups}

Finally we consider the case when $G$ is compact and semisimple, but not necessarily simply connected. For simplicity we only discuss the proof of Formula 1, the other formulas can be proved in completely the same way. 
Let $\pi_{1}(G)$ denote the fundemental group of $G$ which, since $G$ is semisimple, is finite. Let $G^{\prime}$ be the universal covering of $G$. Let $H^{\prime}(t, y, x)$ be the heat kernel of $G^{\prime}$, then the heat kernel on $G$ is given by

$$
H(t, y, x)=\frac{1}{\left(\# \pi_{1}(G)\right)^{2}} \sum_{\gamma \in \pi_{1}(G)} H^{\prime}(t, y, \gamma x),
$$

where the $x, y$ on the left hand side are correspondingly the lifts onto $G^{\prime}$ of $x, y \in G$. Here the reason that we divide by $\left(\# \pi_{1}(G)\right)^{2}$ is due to that fact that $H^{\prime}(t, y, x)$ is not normalized, it should be divided by the volume of $G^{\prime}$.

Consider $f: G^{2 g} \rightarrow G$ and $\mathcal{M}_{u}^{\prime}=f^{-1}(u) / G$ where $f$ is the same as in the simply connected case and $u \in Z(G)$ is a regular value of $f$. Let $\tilde{f}: G^{\prime 2 g} \rightarrow G^{\prime}$ be a lift of $f$. Since $\operatorname{Vol}\left(G^{\prime}\right)=\# \pi_{1}(G) \operatorname{Vol}(G)$, by using Lemma 2 we get

$$
\begin{aligned}
{\left[\# \pi_{1}(G)\right]^{2 g} \int_{G^{2 g}} H(t, f(h), u) d \mathrm{vol} } \\
=\frac{1}{\left(\# \pi_{1}(G)\right)^{2}} \sum_{\gamma \in \pi_{1}(G)} \int_{G^{\prime 2 g}} H^{\prime}(t, \tilde{f}(h), \gamma u) d \mathrm{vol} \\
=\frac{\operatorname{Vol}\left(G^{\prime}\right)^{2 g}}{\left(\# \pi_{1}(G)\right)^{2}} \sum_{\gamma \in \pi_{1}(G)} \sum_{\lambda \in P_{+}} \frac{\chi_{\lambda}\left((\gamma u)^{-1}\right)}{d_{\lambda}^{2 g-1}} e^{-p_{c}(\lambda) t} \\
=\frac{\operatorname{Vol}\left(G^{\prime}\right)^{2 g}}{\# \pi_{1}(G)} \sum_{\lambda \in P_{+}} \frac{\chi_{\lambda}\left(u^{-1}\right)}{d_{\lambda}^{2 g-1}} e^{-p_{c}(\lambda) t} .
\end{aligned}
$$

Here we have used the fact that

$$
d_{\lambda} \chi_{\lambda}\left((\gamma u)^{-1}\right)=\chi_{\lambda}\left(u^{-1}\right) \chi_{\lambda}\left(\gamma^{-1}\right)
$$

and that

$$
\frac{1}{d_{\lambda}} \sum_{\gamma \in \pi_{1}(G)} \chi_{\lambda}\left(\gamma^{-1}\right)
$$

is zero except when $\chi_{\lambda}$ is trivial, in which case it is equal to $\# \pi_{1}(G)$.

On the other hand when $t \rightarrow 0^{+}$, the same method as in the simply connected case gives us

$$
\begin{aligned}
\lim _{t \rightarrow 0^{+}} \int_{G^{2 g}} H(t, f(h), u) d \mathrm{vol} & =\frac{\operatorname{Vol}(G)^{2}}{\# Z(G)} \int_{\mathcal{M}_{u}^{\prime}} \tau\left(\mathcal{C}_{a}\right) \\
& =(2 \pi)^{\operatorname{dim} \mathcal{M}_{u}^{\prime}} \frac{\operatorname{Vol}(G)^{2}}{\# Z(G)} \int_{\mathcal{M}_{u}^{\prime}} e^{\omega_{u}^{\prime}}
\end{aligned}
$$


where $\omega_{u}^{\prime}$ is the induced symplectic form on $\mathcal{M}_{u}^{\prime}$ from the $\omega$ in (1), and $\mathcal{C}_{a}$ is the the same complex as in Lemma 4. By putting the above two formulas together, we get

$$
\int_{\mathcal{M}_{u}^{\prime}} e^{\omega_{u}^{\prime}}=\frac{\# Z(G)}{\# \pi_{1}(G)}\left[\frac{\operatorname{Vol}(G)}{(2 \pi)^{\operatorname{dim} G}}\right]^{2 g-2} \sum_{\lambda \in P_{+}} \frac{\chi_{\lambda}\left(u^{-1}\right)}{d_{\lambda}^{2 g-1}} .
$$

The other formulas can be extended to the non-simply connected case in the same way.

We note that, for $G=S U(2)$, a proof of Formula 3 was announced in [JK]. Their method is completely different from the one used in this paper.

\section{Acknowledgements}

I would like to thank S. Chang and W. Zhang for many interesting discussions. Without them this paper may have never appeared in this form. In June 23 of 1996, I have reported this work in the International Conference on Geometry and Analysis, organized by Prof. S. T. Yau and Prof. S. C. Chang in Tsinghua University, Taiwan. I want to thank Tsinghua University, especially Prof. S.-J. Kao for their hospitality, and the audience for their interests in this work. Bong Lian has also made some very useful comments on an early version.

\section{References}

[AB] M. Atiyah and R. Bott, The Yang-Mills equations over Riemann surfaces, Phil. Trans. Roy. Soc. London A308 (1982), 523-615.

[BD] T. Brocker, T. tom Dieck, Representations of compact Lie groups, SpringerVerlag, 1985.

[BGV] N. Berline, E. Getzler, M. Vergne, Heat kernels and Dirac operators, Grundlehren der Math. Wiss. 298, Springer-Verlag, Berlin, 1992.

[BZ] J.-M. Bismut and W. Zhang, An extension of a theorem by Cheeger and Muller, Astérisque 205 (1992).

[Ch] S. Chang, to appear.

[Ch] _ Nonabelian theta-functions, geometric quatization and a proof of the Verlinde formula, MIT preprint, (1992).

[D] S. K. Donaldson, Gluing techniques in the cohomology of moduli spaces, in Topological metheods in modern mathematics, Publish or Perish, 1995.

[Fa] M. Farber, Combinatorial invariants computing the Ray-Singer analytic torsion, Preprint, (1996).

[Fe] H. Fegan, Introduction to compact Lie groups, Series in Math., 13 World Scientific 1991.

[Fo] R. Forman, Small volume limits of $2-d$ Yang-Mills, Comm. Math. Phys. 151 (1993), 39-52.

[J] L. Jeffrey, Extended moduli spaces of flat connections on Riemann surfaces, Math. Ann. 298 (1994), 667-692. 
[JK] L. Jeffrey and F. Kirwan, Intersection pairings in moduli spaces of holomorphic bundles on a Riemann surfaces, Elec. Res. Ann. Amer. Math. Soc., 1 (1995), 57-71.

[KS] M. Kreck and S. Stolz, Nonconnected moduli spaces of positive sectional curvature metrics, J. Amer. Math. Soc. 6 (1993), 825-850.

[Mi] J. Milnor, A duality theorem for Reidemeister torsion, Ann. of Math. 76 (1962), 137-147.

[Mo] K. Mohri, Residues and topological Yang-Mills theory in two dimensions, Preprint, 1995.

[Liu] K. Liu, Remarks on nonabelian localization formulas, Int. Math. Res. Not. 13 (1995), 683-691.

[Lu] W. Luck, Analytic and topological torsion for manifolds with boundary and symmetry, J. Diff. Geom. 37 (1993), 263-322.

[RS] D. Ray and R. Singer, R-torsion and the Laplacian on Riemannian manifolds, Adv. Math. 7 (1971), 145-210.

[Sz] A. Szenes, The combinatorics of the Verlinde formulas, Symposium on vector bundles in algebraic geometry, (Durham, 1993), London Math. Soc. Lecture Note Ser. 208, Cambridge Univ. Press, 1995.

[U] H. Urakawa, The heat equation on compact Lie group, Osaka J. Math. 12 (1975), 285-297.

[V] M. Vergne, A note on the Jeffrey-Kirwan-Witten localization formula, Topology 35 (1996), 243-266.

[W] E. Witten, On Quantum gauge theory in two dimensions, Comm. Math. Phys. 141 (1991), 153-209.

[W1] _ _ Two-dimensional gauge theory revisited, J. Geom. Phys. 9 (1992), 303368.

Department of Mathematics, Stanford University, Stanford, CA 94305

E-mail address: kefeng@math.stanford.edu 